\title{
Impacto de los procesos de investigación en los procesos de formación humana integral para la Facultad de Ciencias y Tecnologías de la VUAD ${ }^{1}$
}

Impact of the research processes in the processes of integral human formation for the Faculty of Sciences and Technologies of the V U A D

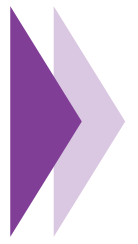

Julia Isabel Roberto

Investigadora principal.

Lingüista de la Universidad Nacional de Colombia. Especialista en Entornos Virtuales de Aprendizaje de la Universidad de Panamá. Magíster en Entornos Virtuales de Aprendizaje de la Universidad de Panamá. Estudiante $2^{\circ}$ semestre del Doctorado en Teoría Crítica en 17, Instituto de Estudios Críticos. Correo electrónico: juliaroberto1978@gmail.com

Wilma E. Lima - Ravelo

Universidad Santo Tomás.

Correo electrónico: wilmalima@ustadistancia.edu.co

Paola A. Sánchez - Hernández

Universidad Santo Tomás.

Correo electrónico: paolasanchez@ustadistancia.edu.co

1. Proyecto de investigación realizado en el marco de la convocatoria 10-2015 del Centro de investigaciones VUAD, Universidad Santo Tomás, perteneciente a la Línea medular Línea Alberto Magno: Ciencia, Tecnología y Medio Ambiente, Línea activa Gestión en Ciencia, Innovación, Tecnologías y Organizaciones. 


\section{Resumen}

La Facultad de Ciencias y Tecnologías (FCT) inició en el año 2014 un proceso de restructuración del área de investigación, revisando e interrelacionando los espacios académicos que comprendían los módulos de investigación para unificar su denominación en todos los programas de pregrado de la Facultad; así mismo, unificar los contenidos, el número de créditos y su orientación; esto debido a que las cátedras con el componente investigativo eran transversales a todos los programas; por tanto se propuso la unión de los elementos mencionados dando lugar a una línea común y espacio académico que facilitara hablar un lenguaje general conceptual a nivel de Investigación Formativa en la Facultad, aprovechando que ya a esa fecha se desarrollaban actividades de investigación formativa.

A partir de lo expuesto, surgió el interés de recopilar y analizar cómo se estaba realizando el proceso investigativo en los programas académicos de la FCT; así como conocer el impacto de las propuestas de investigación que se habían llevado a cabo; su contribución a la formación humana e integral de los estudiantes; su coherencia con los campos de estudio de los programas académicos en el período 2005 y 2014 y así conocer la evolución de la investigación en la Facultad de Ciencias y Tecnologías durante este período.

Palabras clave: investigación, formación humana integral, programas académicos.

\section{Abstract}

The Faculty of Science and Technology (FCT) started in 2014 a restructuring process of the research area, reviewing and interrelated the academic spaces that included the research modules to unify their denomination in all the undergraduate programs of the Faculty; likewise, to unify the contents, the number of credits and their orientation; This is because the chairs with the research component were transversal to all the programs; therefore, the union of the mentioned elements was proposed, giving rise to a common line and academic space that would facilitate speaking a general conceptual language at the level of Formative Research in the Faculty, taking advantage of the fact that at that date formative research activities were developed.

From the above, the interest arose to collect and analyze how the research process was being carried out in the academic programs of the FCT; as well as knowing the impact of the research proposals that had been carried out; its contribution to the human and integral formation of the students; its coherence with the fields of study of academic programs in the period 2005 and 2014 and thus know the evolution of research in the Faculty of Science and Technology during this period.

Keywords: research, comprehensive human education, academic programs. 


\section{Introducción}

La ley 115/94 establece que las instituciones de educación superior (IES) deben incluir en el Proyecto Educativo Institucional - PEI la interrelación con los diferentes actores externos e internos, la relación de la docencia, investigación y proyección social, lo que ha convertido la investigación en un pilar de las IES, desde el cual se apalancan avances en el proceso formativo en los diferentes programas académicos; y específicamente en la Universidad Santo Tomás un componente fundamental que evidencia los avances de los diferentes programas académicos.

En coherencia con lo anterior, la Universidad Santo Tomás (USTA), en su PEI "se ha comprometido con la investigación como fundamento de su compromiso con la sociedad [...] Por ello, las políticas de investigación y docencia [...] están orientadas por su filosofía al desarrollo científico, cultural, social, económico, político y ético, a nivel nacional y latinoamericano". (Universidad Santo Tomás PEI, 2004, p. 132); así mismo, "la Vicerrectoría de la Universidad Abierta y a Distancia (VUAD) se apropia de la función investigación promocionando y fortaleciendo sus procesos dentro de los programas académicos para mejorar impactos que el plan de desarrollo se propuso siguiendo las directrices enunciadas en el Proyecto Investigativo Institucional de la USTA (PROIN)". (Vicerrectoría Universidad Abierta y a Distancia - Universidad Santo Tomás, s.f.).

De forma particular, desde la Facultad de Ciencias y Tecnologías, adscrita a la Vicerrectoría de la Universidad Abierta y a Distancia (VUAD) de la Universidad Santo Tomás
(USTA) (actualmente División de Educación Abierta y a Distancia), dando continuidad a los lineamientos investigativos institucionales, a través de sus programas académicos de grado y postgrado, propuso una estructuración de la investigación, sustentado en el hecho de que los espacios académicos que orientan el componente investigativo carecían de unificación en cuanto a contenidos, número de créditos, denominación y orientación que se proponen; de forma particular se encontró que el caso del espacio "Metodología de la investigación" que es transversal a todos los programas académicos, se unificó respecto a los aspectos mencionados desde el segundo semestre de 2014, y dio lugar a la creación de un segundo espacio que facilita la introducción completa de las bases conceptuales que permiten iniciar procesos de investigación, buscando la articulación entre la formación y la investigación.

En segundo lugar, los programas académicos de la Facultad, actualmente, adelantan un ejercicio de creación y consolidación de sus líneas de investigación, con el fin de orientar sus acciones investigativas, para de esta forma también articular los procesos de formación con la investigación en sentido estricto.

De otro lado, existen programas académicos que desarrollan actividades de investigación formativa, expresadas en semilleros de investigación consolidados; sin embargo, existen otros programas académicos que están iniciando hasta ahora este proceso.

De acuerdo a lo expuesto hasta aquí, surge el interés por determinar cómo se desarrollan los procesos de investigación en los programas académicos de la Facultad de Ciencias y Tecnologías, en este sentido es importante 
identificar los parámetros, las condiciones y los aportes que brindan dichos procesos a la formación académica específica, de igual manera, conocer cómo las propuestas de investigación que se han llevado a cabo contribuyen a la formación humana e integral de los estudiantes y establecen relación con los campos de estudio de los programas, lo que permitirá respaldar y consolidar los procesos investigativos.

De acuerdo a esto, se adelanta una aproximación al reconocimiento del impacto que se ha logrado desde los procesos de investigación en la formación humana e integral de los estudiantes y su coherencia con los campos de estudio de los programas académicos de la Facultad de Ciencias y Tecnologías en el período 2005 y 2014 para conocer la evolución de la investigación durante este período.

\section{El proceso}

Con el fin de conocer esta evolución, durante el primer semestre del 2015, se revisaron los campos de estudio o énfasis de los programas académicos de la Facultad de Ciencias y Tecnologías VUAD, a saber: administración ambiental y de los recursos naturales, administración de empresas agropecuarias, zootecnia, administración de empresas, ingeniería en informática, tecnología en logística de operaciones y construcción en arquitectura e ingeniería.

En el segundo semestre de 2015 se realizaron visitas a los CAU Villavicencio, Bucaramanga, Tunja, Medellín, Barranquilla, Facatativá, Montería, Armenia y Cali, con el propósito de conocer los diferentes procesos de investigación realizados en cada uno de ellos entre los años 2005 y 2014; proceso que incluyó la revisión de los trabajos de grado que los estudiantes realizaron durante el periodo de estudio para establecer su relación con la formación humana-integral.

En cada CAU se aplicaron tres instrumentos: matriz para programas académicos, matriz para CAU y entrevista semiestructurada que contenía seis preguntas abiertas, aplicado directamente a los docentes con funciones de investigación en los programas de la Facultad que ofertaba cada CAU visitado, las preguntas realizadas fueron las siguientes:

1. ¿Los procesos de investigación tienen relación con las áreas de estudio del programa académico al que pertenece?

2. ¿Las investigaciones que se han llevado a cabo en el marco de proyectos, de semilleros de investigación y de trabajos de grado desarrollan sólo temas disciplinares?

3. ¿Las investigaciones que se han desarrollado en la Facultad tienen relación con la formación integral expuesta en el Modelo Educativo Pedagógico de la Universidad? ¿En qué aspectos?

4. ¿El desarrollo de procesos de investigación (proyectos, semilleros, trabajos de grado) es objeto de interés tanto para los estudiantes como para los docentes del CAU?

5. ¿Qué actividades, estrategias o elementos se deben considerar para promover 
los procesos de investigación tanto en los estudiantes como en los docentes?

6. A partir de un proceso de investigación (proyectos, semilleros, trabajos de grado) describa los aportes a la formación de los estudiantes, al ejercicio docente y a la disciplina.

Finalmente, se utilizó el software Atlas.TI para sistematizar los datos obtenidos, lo que permitió correlacionar las variables establecidas para la investigación (proyectos, semilleros, trabajos de grado / campos de estudio y, formación humana-integral).

\section{Resultados}

Como resultado del ejercicio expuesto, se logró identificar los campos de acción de estudio de cada uno de los programas académicos de la Facultad de Ciencias y Tecnologías, estableciendo la relación entre éstos y como se estaban aplicando en los trabajos de grado que los estudiantes realizaron durante el periodo de estudio, para establecer su relación con la formación humana-integral; se estableció la relación con la formación humana e integral identificando los puntos a fortalecer en el proceso de investigación.

De proyectos encontrados en los CAU, en general se encontraron procesos sin el año de elaboración y con muy pocos registros en el periodo de estudio 2011 a 2014 así: CAU Villavicencio: 11 proyectos orientados a procesos de pasantías, trabajos monográficos y trabajos de grado, CAU Bucaramanga: 26 proyectos; CAU Tunja: 2 proyectos; CAU Barranquilla: 13 proyectos; CAU Armenia: 29 proyectos; CAU Medellín: 28 proyectos; CAU Montería: 21 proyectos; CAU Facatativá: 8 proyectos; CAU Cali: 13 proyectos.

\section{Conclusiones}

De acuerdo con la información que se recolectó para el periodo 2005 a 2014 en las visitas a los CAU, se encontró que los programas académicos de la Facultad de Ciencias y Tecnologías desarrollaron procesos investigativos de aplicación disciplinar en las regiones, los cuales se enfocaron en problemáticas del entorno, siendo las prácticas administrativas las más frecuentes frente a los trabajos de grado y pasantías.

En los CAU que se visitaron, se evidenció la falta de una base de datos que recopile la información de los trabajos de grado, las prácticas administrativas, las pasantías, entre otros, que realizan los estudiantes desde los programas académicos de la Facultad; situación que evidencia el deterioro del proceso formativo en investigación, la falta de continuidad en la línea que cada programa se ha propuesto seguir y la no visibilidad del trabajo desarrollado por los mismos programas.

Con la aplicación de las entrevistas, se encontró un gran número de personas que eligen cursar especializaciones como opción de grado para obtener el título de pregrado, lo que genera una notoria disminución en la elaboración de monografías, la participación en proyectos de investigación o semilleros

Los docentes de los CAU visitados manifiestan también la necesidad de fortalecer la 
formación en investigación tanto para estudiantes como docentes que se han vinculado a los semilleros de investigación, así mismo se requiere hacer acompañamiento desde el Centro de Investigaciones en Bogotá y la Facultad para consolidar e incentivar este proceso de tal manera que los estudiantes hagan el recorrido desde el semillero de investigación hasta la opción de grado.

Finalmente, es importante destacar que los proyectos registrados en cada CAU, denotan la formación humana integral de los estudiantes, "en la que el desarrollo de todas las dimensiones de la vida personal genera que los estudiantes adquieran una conciencia superior que les permita comprender su propio valor histórico, su pro $\neg$ pia función en la vida, sus propios derechos y deberes, y los haga capaces de inter $\neg$ venir y participar lúcida y responsablemente en la vida social, cultural, económica y política, aportando su actitud creativa y su aptitud crítica e investigativa" (Universidad Santo Tomás MEP, 2010).

\section{Referencias}

Centro de investigación VUAD-USTA. (2014). Lineamientos de investigación. Centro de investigación VUAD-USTA. Bogotá: http:// unidadinvestigacion.usta.edu.co/images/ documentos/investigacion.pdf

Universidad Santo Tomás. (2009). Política de Investigación. Bogotá: USTA.

Universidad Santo Tomás. (2010). Modelo Educativo Pedagógico. Bogotá: USTA.
Universidad Santo Tomás PEI. (2004). Proyecto Educativo Institucional (PEI) USTA. Bogota: USTA. Recuperado de: http://www.usta. edu.co/images/documentos/documentosinstitucionales/pei.pdf

Vicerrectoría Universidad Abierta y a Distancia - Universidad Santo Tomás. (s.f.). Centro de Investigación VUAD. Recuperado de: https://www.ustadistancia.edu.co/ index.php/centro-de-investigacion-vuad

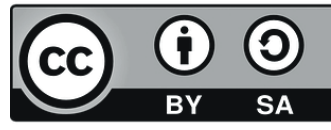

